\title{
Glicerina bruta na dieta de vacas lactantes mantidas em pastagem tropical: comportamento ingestivo
}

\author{
Crude glycerin in diets for lactating cows grazing on tropical \\ pasture: ingestive behavior
}

\author{
Hermógenes Almeida Santana Junior ${ }^{1 *}$; Mauro Pereira Figueiredo²; Elizangela \\ Oliveira Cardoso Santana; ; Fabricio Bacelar Lima Mendes'; George Abreu Filho ${ }^{3}$; \\ Alyson Andrade Pinheiro4; Mateus Melo Lisboa ${ }^{3}$; Yann Santos Luz ${ }^{3}$; Pablo Teixeira \\ Viana $^{3}$; Antônio Hosmylton Carvalho Ferreira ${ }^{1}$; Carmen Lúcia de Souza Rech ${ }^{5}$
}

\section{Resumo}

\begin{abstract}
Objetivou-se com este estudo avaliar o comportamento ingestivo de vacas lactantes mantidas em pastagem tropical suplementadas com diferentes níveis de glicerina bruta na dieta. Foram utilizadas 10 vacas lactantes, 3/4 Holandesa x 1/4 Gir Leiteiro, primíparas, com $109 \pm 24$ dias de lactação, idade média de $30 \pm 6$ meses e peso corporal médio de 426,2 $\pm 68,29 \mathrm{~kg}$, distribuídas em cinco tratamentos, utilizando-se dois quadrados latinos cinco por cinco (5x5), Os tratamentos foram constituídos pelos níveis de inclusão de glicerina bruta na dieta $(0 ; 4,5 ; 9 ; 13,5$ e $18 \%$; base matéria seca). A avaliação do comportamento foi realizada no $14^{\circ}$ dia de cada período, sendo feitas observações a cada cinco minutos por um período de 24 horas. Os resultados foram analisados estatisticamente por meio de análises de variância e regressão a 5\% de probabilidade. Para os tempos de pastejo, outras atividades, ruminação, alimentação no cocho, número de períodos de pastejo, outras atividades, ruminação e alimentação no cocho, assim como taxa de bocado, número de mastigações merícicas e tempo por bolo alimentar, e as eficiências de alimentação e ruminação não foram verificadas diferenças entre os níveis de glicerina bruta na dieta $(\mathrm{P}>0,05)$. A inclusão de glicerina bruta na dieta de vacas lactantes em pastagens tropicais não promove variações no comportamento ingestivo. A ausência de efeitos negativos relacionados ao uso deste coproduto em substituição ao milho nestas condições permite a sua utilização em até $18 \%$ de glicerina bruta em dietas para vaca leiteiras sem alterar os parâmetros comportamentais relacionados à ingestão de alimentos.
\end{abstract}

Palavras-chave: Bocado, coproduto, glicerol, pastejo, produção de leite

\begin{abstract}
The objective of this study was to evaluate the ingestive behavior of lactating dairy cows grazing on tropical pasture supplemented with different levels of crude glycerin in the diet. Ten lactating cows, $3 / 4$ Gir x $1 / 4$ Holstein Dairy cows, primiparous, and $109 \pm 24$ days of lactation, mean age $30 \pm 6$ months and mean body weight of $426.2 \pm 68.29 \mathrm{~kg}$, distributed into five treatments, in double (5x5) Latin square design, were used. The treatments consisted of inclusion levels of crude glycerin in the diet
\end{abstract}

\footnotetext{
${ }^{1}$ Profs. Assistentes, Universidade Estadual do Piauí, Corrente, PI. E-mail: hsantanajunior@hotmail.com; hosmylton@hotmail.com

${ }^{2}$ Prof. Pleno, Universidade Estadual do Sudoeste da Bahia, UESB, Vitória da Conquista, BA. E-mail: mfigue2@yahoo.com.br

${ }^{3}$ Discente(s) de Pós-graduação, UESB, Itapetinga, BA. E-mail: eocardoso@hotmail.com; fabricio.bacelar@hotmail.com; georgeabreu16@hotmail.com; lisboazoot@hotmail.com; yann_agronomia@yahoo.com.br; pabloteixeira@hotmail.com

${ }^{4}$ Discente de Pós-Doutorado, EMATER/GO, Goiânia, GO. E-mail: jagualyson@bol.com.br

${ }^{5}$ Prof ${ }^{\text {a }}$ Plena, UESB, Itapetinga, BA. E-mail: clrech@bol.com.br

* Autor para correspondência
} 
$\left(0.0,4.5,9.0,13.5\right.$, and $18.0 \%$ dry matter basis). The behavior evaluation was performed on the $14^{\text {th }}$ day of each period, with observations every five minutes for a period of 24 hours. The results were analyzed statistically using analysis of variance and regression at $5 \%$ probability. For the grazing time, other activities, rumination, feeding trough, the number of grazing periods, other activities, rumination and feeding trough, as well as bit rate, number and time of chews per bolus, and efficiencies of eating and ruminating were not significant differences among crude glycerin levels in the diet $(\mathrm{P}>0.05)$. The inclusion of crude glycerin in diets for lactating cows in tropical pastures does not promote changes in ingestive behavior. The absence of negative effects related to the use of this byproduct in substitution of corn in these conditions allows its use in up to $18 \%$ crude glycerin in diets for dairy cows without changing the behavioral parameters related to food intake.

Key words: Bit, byproduct, glycerol, grazing, milk production

\section{Introdução}

A glicerina é produzida da transesterificação de óleos vegetais que reagindo com um álcool (metanol ou etanol) e, catalisada por uma base, formando ácidos graxos metílicos (THOMPSON; HE, 2006) na produção de biocombustíveis. Porém, como todo coproduto, a mesma apresenta variações na qualidade, podendo conter teores de glicerol superiores ou inferiores a 95\% (purificada e bruta, respectivamente).

O aumento de produção do biodiesel acarreta em elevação da disponibilidade de glicerina, tornando-a um coproduto disponível para alimentação de bovinos (OSBORNE et al., 2009; NUNES et al., 2010). Devido aos preços reduzidos, pode substituir concentrados energéticos na alimentação tornando-a atrativa financeiramente.

Vários estudos descreveram a utilização de variáveis comportamentais para nortear a avaliação de dietas (ALLDEN; WHITTAKER, 1970; MISSIO et al., 2010; PEREIRA et al., 2011; SANTANA JÚNIOR et al., 2013b; SANTANA JÚNIOR et al., 2013c). Paralelamente, a ingestão de matéria seca pelas vacas mantidas em pastagens tropicais se constitui em um dos principais fatores que limitam a produção de leite (FUKUMOTO et al., 2010). Neste contexto, a inclusão da glicerina bruta na dieta pode modificar o comportamento ingestivo dos ruminantes, notadamente em razão de algumas das suas características físicas e químicas. A sua apresentação na forma líquida à temperatura ambiente, como também o seu caráter lipídico, se constituem em exemplos destas características (SANTANA JÚNIOR et al., 2013a).

Tendo em vista a escassez de trabalhos que abordam a utilização deste coproduto na dieta de ruminantes em pastagens, a avaliação do comportamento ingestivo pode contribuir para o melhor entendimento do uso deste composto para vacas leiteiras.

Objetivou-se com este estudo, avaliar o comportamento ingestivo de vacas lactantes mantidas em pastagem tropical suplementadas com diferentes níveis de glicerina bruta na dieta.

\section{Material e Métodos}

O experimento foi conduzido na Fazenda Rancho Santana, situada em Jequié/BA, localizada a $13^{\circ} 52^{\prime}$ 14 " de latitude Sul, $40^{\circ} 09^{\prime}$ 47" de longitude Oeste, com altitude média de $260 \mathrm{~m}$. O trabalho de campo foi realizado de 21 de dezembro de 2010 a 16 de março de 2011, implantado numa área de 7600 $\mathrm{m}^{2}$, dividida em 14 piquetes de aproximadamente $380 \mathrm{~m}^{2}$ cada, formada com Brachiaria brizantha cultivar MG-5, sendo irrigada com aspersão em malha e adubada durante o período experimental com $240 \mathrm{~kg}$ de N/ha na forma de ureia. Foram coletados os dados de temperatura, precipitação e irrigação durante o período experimental (Tabela 1). Foi utilizado o sistema de ocupação rotacionada de piquetes com ocupação de um dia e 13 dias de descanso. 
Foram utilizadas 10 vacas lactantes, $3 / 4$ Holandesa x 1/4 Gir Leiteiro, primíparas, com $109 \pm 24$ dias de lactação, idade média inicial de $30 \pm 6$ meses e peso corporal inicial médio de 426,2 $\pm 68,29 \mathrm{~kg}$ sendo distribuídas em cinco tratamentos, em duplo quadrado latino cinco por cinco $(5 \times 5)$. Estes animais foram suplementados com alimentos concentrados para atender as exigências de mantença, crescimento $(\mathrm{GPC}=300 \mathrm{~g} / \mathrm{dia})$ e produção de 12 litros de leite a 3,5\% gordura (NRC, 2001). As dietas foram balanceadas para serem isoenergéticas $(60,0 \%$ NDT) e isoproteicas $(14,4 \% \mathrm{~PB})$.

Tabela 1. Temperatura média, precipitação total e irrigação total durante cada período experimental e a média dos períodos.

\begin{tabular}{lcccccc}
\hline \multirow{2}{*}{\multicolumn{1}{c}{ Item }} & \multicolumn{5}{c}{ Período } & \multirow{2}{*}{ Média } \\
\cline { 2 - 5 } & $1^{\mathbf{0}}$ & $2^{\mathbf{o}}$ & $3^{\mathbf{o}}$ & $4^{\mathbf{0}}$ & $5^{\mathbf{0}}$ & \\
\hline Tmín. à sombra & 23,7 & 22,0 & 22,9 & 24,1 & 22,9 & 23,1 \\
Tmáx. à sombra & 35,4 & 34,7 & 37,7 & 41,7 & 37,7 & 37,4 \\
Tmín. ao sol & 21,8 & 20,0 & 20,3 & 21,7 & 20,5 & 20,9 \\
Tmáx. ao sol & 37,8 & 37,1 & 40,1 & 43,1 & 42,2 & 40,1 \\
Precipitação (mm) & 33 & 13 & 52 & 0 & 157 & 51 \\
Irrigação (mm) & 170 & 165 & 152 & 180 & 20 & 137 \\
\hline
\end{tabular}

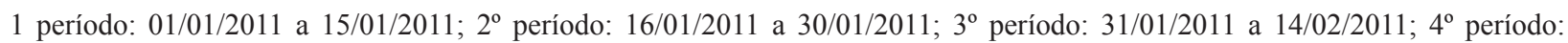
15/02/2011 a 01/03/2011; $5^{\circ}$ período: 02/03/2011 a 16/03/2011.

Tmín.- temperatura mínima em graus Celsius; Tmáx.- temperatura máxima em graus Celsius

Fonte: Elaboração dos autores.

Os tratamentos foram constituídos de diferentes níveis de glicerina na dieta: G0 - Dieta com $0 \%$ de inclusão da glicerina na matéria seca total (controle); G4,5 - Dieta com 4,5\% de inclusão da glicerina na matéria seca total (MST); G9 - Dieta com 9\% de inclusão da glicerina na MST; G13,5 - Dieta com $13,5 \%$ de inclusão da glicerina na MST; G18 Dieta com $18 \%$ de inclusão da glicerina na MST. Estimou-se uma relação volumoso:concentrado de $75: 25$, sendo que a inclusão da glicerina representou até $39 \%$ do concentrado no tratamento G18.

$\mathrm{O}$ manejo diário das vacas iniciou-se às cinco horas, quando retornavam da pastagem para a realização da primeira ordenha e, a segunda ordenha, era iniciada às 16 horas. A ordenha foi realizada de forma mecânica (tipo balde ao pé, modelo fila indiana com fosso). O suplemento com concentrados (Tabela 2) foi fornecido logo após as ordenhas, de forma individual em cocho de polipropileno, com disponibilidade de $100 \mathrm{~cm}$ lineares por animal.

O experimento teve duração de 75 dias, divididos em cinco períodos de 15 dias, sendo 10 dias para adaptação dos animais às dietas experimentais e cinco dias para coleta de dados.

A pastagem foi avaliada diariamente, utilizando o método de McMeniman (1997), Wilm, Costello e Klipple (1994), Gardner (1986) e Campbell (1966), para a coleta, biomassa residual diária (BRD), biomassa de forragem (disponibilidade) e taxa de acúmulo diário (TAD), respectivamente (Tabela 3). 
Tabela 2. Proporções dos ingredientes do concentrado, na base da matéria natural.

\begin{tabular}{lccccc}
\hline \multirow{2}{*}{\multicolumn{1}{c}{ Ingrediente (\%) }} & \multicolumn{5}{c}{ Nível de glicerina bruta na dieta (\%) } \\
\cline { 2 - 6 } & $\mathrm{G} 0$ & $\mathrm{G} 4,5$ & $\mathrm{G} 9$ & $\mathrm{G} 13,5$ & $\mathrm{G} 18$ \\
\hline Milho grão moído & 77,20 & 65,29 & 53,14 & 40,75 & 28,11 \\
Farelo de soja & 20,97 & 22,42 & 24,91 & 27,45 & 30,04 \\
Glicerina bruta & --- & 9,45 & 19,09 & 28,91 & 38,92 \\
Calcário calcítico $_{\text {Fosfato bicálcico }}$ & 0,86 & 0,86 & 0,86 & 0,86 & 0,82 \\
Sal mineral $^{1}$ & --- & --- & --- & --- & 0,07 \\
\hline
\end{tabular}

${ }^{1}$ Composição: Cálcio 200 g; fósforo 100 g; sódio 68 g; magnésio 15 g; enxofre 12 g; cobalto 200 mg; cobre 1650 mg; iodo 195 mg; manganês 1960 mg; níquel 40 mg; selênio 32 mg; zinco 6285 mg; ferro 560 mg; flúor (máximo) 1000 mg.

Fonte: Elaboração dos autores.

Tabela 3. Média e desvio-padrão (DP) das características da forragem durante o período experimental.

\begin{tabular}{cccccccccccc}
\hline & $\mathrm{DTMS}^{1}$ & $\mathrm{DMSV}^{1}$ & $\mathrm{MSpd}^{2}$ & $\mathrm{BRD}^{3}$ & $\mathrm{TL}^{4}$ & $\mathrm{TAD}^{3}$ & $\mathrm{OF}^{5}$ & $\mathrm{AF}^{6}$ & $\mathrm{~F}^{2}$ & $\mathrm{C}^{2}$ & $\mathrm{MM}^{2}$ \\
\hline Média & 5367 & 4187 & 72 & 1945 & 12,5 & 263 & 39 & 52 & 38 & 52 & 10 \\
DP & \pm 1243 & \pm 745 & \pm 5 & \pm 206 & $\pm 0,2$ & \pm 47 & \pm 8 & \pm 2 & \pm 5 & \pm 6 & \pm 2 \\
\hline
\end{tabular}

${ }^{1}$ Expresso em kg/ha; ${ }^{2}$ Expresso em \%; ${ }^{3}$ Expresso em kg MS/ha/dia; ${ }^{4}$ Expresso em UA/ha; ${ }^{5}$ Expresso em kg MS/100 kg PC/dia; ${ }^{6}$ Expresso em $\mathrm{cm}$.

DTMS - disponibilidade total de matéria seca; DMSV - disponibilidade de matéria seca verde; MSpd -matéria seca potencialmente digestível; BRD - biomassa residual diária; TL - taxa de lotação; TAD - taxa de acúmulo diário; OF - oferta de forragem; AF altura da forragem; F - folha; C - colmo; MM - matéria morta.

Fonte: Elaboração dos autores.

A taxa de lotação (TL) foi calculada considerando a unidade animal (UA, $450 \mathrm{~kg}$ PC). A oferta de forragem (OF) foi calculada de acordo com a seguinte fórmula: $\mathrm{OF}=\{(\mathrm{BRD} *$ área $+\mathrm{TAD} *$ área $) /$ $\left.\mathrm{PC}_{\text {total }}\right\} * 100$, em que: $\mathrm{OF}=$ oferta de forragem, em $\mathrm{kg} \mathrm{MS} / 100 \mathrm{~kg} \mathrm{PV} /$ dia; BRD = biomassa residual total, em $\mathrm{kg}$ de $\mathrm{MS} / \mathrm{ha} /$ dia; TAD = taxa de acúmulo diário, em $\mathrm{kg} \mathrm{MS} / \mathrm{ha} / \mathrm{dia} ; \mathrm{PC}=$ peso corporal total dos animais, em $\mathrm{kg} / \mathrm{ha}$.

Foi calculado a MS potencialmente digestível (MSpd) da pastagem, conforme descrito por Paulino; Detmann e Valadares Filho (2006), pela equação: $\mathrm{MSpd}=0,98 *(100-\% \mathrm{FDN})+(\% \mathrm{FDN}$ - \%FDNi). Foi mensurada a altura comprimida da forragem utilizando uma régua graduada, com o auxílio de uma folha plástica transparente, em todos os pontos de coleta de forragem. Durante o período de coleta (últimos cinco dias de cada período) foram coletadas as amostras do pastejo simulado, que foram obtidas por meio de observação dos animais experimentais, identificando-se o tipo de material consumido e coletando-se uma amostra semelhante ao alimento ingerido (JOHNSON, 1978).

Para estimar a produção fecal foi utilizado a LIPE $^{\circledR}$ (Lignina isolada e purificada de eucalipto) como indicador externo, sendo administrada diariamente uma capsula após o fornecimento de concentrado durante sete dias, das quais três dias foram para adaptação e regulação do fluxo de excreção do marcador e quatro dias para coleta de fezes. Para determinação do indicador interno fibra em detergente neutro indigestível (FDNi), as amostras de forragem, de fezes e de concentrados foram incubadas no rúmen de quatro animais fistulados por 240 horas (CASALI et al., 2008), considerando o resíduo como a fração indigestível.

O consumo de MS (Tabela 4) foi obtido por meio da seguinte equação: $\mathrm{CMS}=\{[(\mathrm{PF} * \mathrm{CIFZ})-$ IS]/CIFR $\}+$ CMSS, em que CMS é o consumo de matéria seca (kg/dia); PF é a produção fecal $(\mathrm{kg} /$ 
dia); CIFZ concentração do indicador presente nas fezes $(\mathrm{kg} / \mathrm{kg})$; IS é o indicador presente no suplemento ( $\mathrm{kg} / \mathrm{dia})$; CIFR é a concentração do indicador presente na forragem $(\mathrm{kg} / \mathrm{kg})$ e o CMSS que é o consumo de matéria seca do suplemento $(\mathrm{kg} / \mathrm{dia})$.

As análises laboratoriais foram realizadas no Laboratório de Nutrição Animal do Departamento de Fitotecnia e Zootecnia da Universidade Estadual do Sudoeste da Bahia - UESB, Campus de Vitória da Conquista/BA. As amostras do concentrado, do pastejo simulado (Tabela 5) e das fezes foram présecadas em estufa de ventilação forçada a $55{ }^{\circ} \mathrm{C}$ por 72 horas. O teor de matéria seca, proteína bruta, matéria mineral e extrato etéreo foram analisados de acordo com AOAC (2000). Foram analisadas as fibras em detergente neutro e ácido, pelo método sequencial de Van Soest, Robertson e Lewis (1991), utilizando o analisador de fibras Ankom 200, e corrigido para cinza e proteína de acordo com Hall (2003).

Tabela 4. Consumo diário em vacas lactantes mantidas em pastagem tropical com diferentes níveis de glicerina bruta na dieta.

\begin{tabular}{lrrrrr}
\hline \multirow{2}{*}{ Item } & \multicolumn{5}{c}{ Nível de glicerina bruta na dieta (\%) } \\
\cline { 2 - 5 } & $\mathrm{G} 0$ & $\mathrm{G} 4,5$ & $\mathrm{G} 9$ & $\mathrm{G} 13,5$ & $\mathrm{G} 18$ \\
\hline CMSS (kg) & 4,65 & 4,65 & 4,65 & 4,65 & 4,65 \\
CMSF (kg) & 9,59 & 8,63 & 8,52 & 8,74 & 9,55 \\
CMST (kg) & 14,24 & 13,28 & 13,17 & 13,40 & 14,20 \\
CFDN (kg) & 7,06 & 6,37 & 6,34 & 6,48 & 7,01 \\
CMO (kg) & 13,77 & 12,77 & 12,65 & 12,77 & 13,58 \\
CCNF (kg) & 3,90 & 3,80 & 3,75 & 3,73 & 3,82 \\
CPB (kg) & 2,05 & 1,91 & 1,90 & 1,93 & 2,04 \\
CNDT (kg) & 8,41 & 7,97 & 7,99 & 8,12 & 8,56 \\
\hline
\end{tabular}

CMSS - consumo de MS do suplemento; CMSF - consumo de MS da forragem; CMST - consumo de MS total; CFDN - consumos de fibra em detergente neutro; CMO - consumo de matéria orgânica; CCNF - consumo de carboidratos não fibrosos; CPB consumo de proteína bruta; CNDT - consumo de nutrientes digestíveis totais.

Fonte: Elaboração dos autores.

Tabela 5. Composição bromatológica de amostras da forragem coletada por meio do pastejo simulado nos respectivos períodos experimentais.

\begin{tabular}{|c|c|c|c|c|c|c|}
\hline \multirow{2}{*}{ Item } & \multicolumn{5}{|c|}{ Período } & \multirow{2}{*}{ Média } \\
\hline & $1^{\mathrm{o}}$ & $2^{\circ}$ & $3^{\circ}$ & $4^{\circ}$ & $5^{\circ}$ & \\
\hline $\mathrm{MS}^{1}$ & 29,85 & 32,04 & 30,69 & 25,11 & 32,35 & 30,01 \\
\hline $\mathrm{MO}^{2}$ & 91,67 & 89,64 & 90,65 & 90,73 & 90,92 & 90,72 \\
\hline $\mathrm{FDNcp}^{2}$ & 61,41 & 64,38 & 61,58 & 62,39 & 61,76 & 62,30 \\
\hline $\mathrm{FDA}^{2}$ & 37,40 & 37,77 & 35,58 & 36,60 & 37,63 & 36,99 \\
\hline $\mathrm{PB}^{2}$ & 13,28 & 14,32 & 13,74 & 13,21 & 14,49 & 13,34 \\
\hline $\mathrm{CNF}^{2}$ & 13,96 & 7,00 & 12,86 & 10,90 & 12,72 & 11,49 \\
\hline
\end{tabular}

${ }^{1}$ Expresso em \%; ${ }^{2}$ Expresso em \% da matéria seca.

MS - matéria seca; MO - matéria orgânica; FDNcp - fibra em detergente neutro corrigido para cinzas e proteína; FDA - fibra em detergente ácido $\mathrm{PB}$ - proteína bruta; $\mathrm{CNF}$ - carboidratos não-fibrosos.

Fonte: Elaboração dos autores. 
Os carboidratos não fibrosos (CNF) foram obtidos por intermédio da equação: $100-(\% \mathrm{~PB}+$ $\%$ EE $+\%$ Cinza + \%FDN) (SNIFFEN et al., 1992). Os teores de nutrientes digestíveis totais (NDT) foram obtidos de acordo com Weiss (1999) pela equação: NDT $=$ PBD + FDNcpD + CNFD + (2,25 $x$ EED), em que PBD, FDNcpD, CNFD e EED significam, respectivamente, consumos de $\mathrm{PB}$, FDNcp, CNF e EE digestíveis.

A avaliação do comportamento foi realizada no $14^{\circ}$ dia de cada período, totalizando cinco avaliações, cujas observações foram feitas a cada cinco minutos conforme Gary, Sherritt e Hale (1970), por um período de 24 horas, a fim de identificar o tempo destinado ao pastejo, ruminação, alimentação no cocho e outras atividades. Os animais foram avaliados visualmente, por dois observadores treinados e posicionados estrategicamente de forma a não influenciar o comportamento dos animais. Foram utilizados relógios digitais para cronometrar o tempo utilizado para cada atividade.

As variáveis comportamentais estudadas foram: tempo de pastejo (PAS), tempo de ruminação (RUM), tempo de alimentação no cocho (COC) e tempo em outras atividades (OUT). As atividades comportamentais foram consideradas mutuamente excludentes, conforme definição de Pardo et al. (2003).

O tempo gasto pelos animais na seleção e apreensão da forragem, incluindo os curtos espaços de tempo utilizados no deslocamento para seleção da forragem foi considerado tempo de pastejo. $\mathrm{O}$ tempo de ruminação correspondeu aos processos de regurgitação, remastigação, reinsalivação e redeglutição. O tempo de alimentação no cocho foi o tempo despendido pelo animal no consumo de suplemento, e o tempo em outras atividades (descanso, consumo de água, interações dentre outros), foram todas as atividades com exceção das citadas acima.

O tempo de alimentação total (TAT) e de mastigação total (TMT) foi determinado pelas equações abaixo: TAT $=$ PAS $+\mathrm{COC}$, em que: PAS (minutos) $=$ tempo de pastejo; COC (minutos) $=$ tempo de alimentação no cocho; TMT = PAS + RUM + COC, em que: PAS (minutos) = tempo de pastejo; RUM (minutos) = tempo de ruminação; COC (minutos) $=$ tempo de alimentação no cocho.

A discretização das séries temporais foi realizada diretamente nas planilhas de coleta de dados, com a contagem dos períodos discretos de pastejo, ruminação, outras atividades e alimentação no cocho. A duração média de cada um dos períodos discretos foi obtida pela divisão dos tempos diários de cada uma das atividades pelo número de períodos discretos da mesma atividade. Foram calculados as eficiências de alimentação, em gramas por hora, da MS, FDN, NDT, CNF e PB e eficiência de ruminação da MS e FDN, onde o consumo dos mesmos foi dividido pelo tempo de alimentação total (eficiência de alimentação) ou pelo tempo de ruminação (eficiência de ruminação).

Foram realizadas ainda, observações em dois turnos do dia (manhã e tarde), e com três repetições por período (BURGER et al., 2000), a fim de determinar o número de mastigações merícicas por bolo ruminado (MMB) e o tempo gasto para ruminação de cada bolo (TBo).

As variáveis número de bolo ruminado por dia (BOL), velocidade de mastigação (VeM), tempo por mastigação merícica (TeM) e mastigações merícicas por dia (MMnd) foram calculadas pelas equações abaixo: BOL = RUM / TBo, em que: BOL (número por dia); RUM (segundos/dia) tempo de ruminação; TBo (segundos) - tempo por bolo ruminado; VeM = MMB / TBo, em que: VeM (segundos); MMB - número de mastigações merícicas por bolo; TBo (segundos) - tempo por bolo ruminado; TeM = TBo / $\mathrm{MMB}$, em que: TeM (segundos); TBo (segundos) - tempo por bolo ruminado; MMB - número de mastigações merícicas por bolo; $\mathrm{MMnd}=\mathrm{BOL} * \mathrm{MMB}$, em que: MMnd (número por dia); BOL - número de bolos ruminados por dia; $\mathrm{MMB}$ - número de mastigações merícicas por bolo. 
Durante os mesmos períodos de avaliação do comportamento animal, quando os animais estiveram em atividade de pastejo a mais de 30 minutos, foi registrada a taxa de bocado $(\mathrm{TxB})$ dos animais de cada tratamento, estimada por meio do tempo gasto pelo animal para realizar 20 bocados (HODGSON, 1982). Para o cálculo da massa de bocado em MS ( $\left.\mathrm{MaB}_{\mathrm{MSF}}\right)$ e $\mathrm{MO}\left(\mathrm{MaB}_{\mathrm{MOF}}\right)$, foi dividido o consumo diário de MS da forragem ou o consumo de MO da forragem pelo total de bocados diários (JAMIESON; HODGSON, 1979).

Também foi avaliado o número de bocados e o tempo entre duas deglutições. Os resultados das observações de bocados e deglutição foram registrados em seis ocasiões durante o dia, conforme Baggio et al. (2009), sendo três avaliações durante a manhã e três à tarde, e usados também para determinar o número de bocados por dia (NBD), que é o produto da multiplicação entre taxa de bocado e tempo de pastejo.

Em todas as variáveis comportamentais um animal representou uma unidade experimental. Os resultados foram analisados estatisticamente por meio de análises de variância e regressão a $5 \%$ de probabilidade, com o auxílio do programa SAEG (versão 9.1).

\section{Resultados e Discussão}

Para os tempos de pastejo, outras atividades, ruminação e alimentação no cocho não foram verificadas diferenças entre os níveis de glicerina bruta (GB) na dieta $(\mathrm{P}>0,05)$ (Tabela 6). Na literatura, alguns fatores são citados como responsáveis pelas variações no tempo de pastejo (PAS), como nível de suplemento (MACARI et al., 2007; BREMM et al., 2008), estrutura do dossel forrageiro (REGO et al., 2006; PALHANO et al., 2007; ÍTAVO et al., 2008; BAGGIO et al., 2009; ZANINE et al., 2009; TEIXEIRA et al., 2010, 2011) e teor de fibra em detergente neutro (SANTOS et al., 2006). Zanine et al. (2009) avaliando o comportamento ingestivo de vacas Girolando consumindo duas forrageiras, encontraram média de 562 minutos de pastejo em Brachiaria brizantha. Entretanto, a média encontrada neste estudo foi inferior (446 minutos) a esta, provavelmente devido ao fornecimento de suplemento concentrado, que acarreta em diminuição do tempo de pastejo (BREMM et al., 2008).

A ruminação compreende os processos de regurgitação, remastigação, reinsalivação e redeglutição, sendo estimulada pelo percentual de fibra efetiva na dieta (ALLEN, 1996). A semelhança estatística entre os níveis de glicerina bruta (GB) foi consequência do consumo semelhante de fibra em detergente neutro presente nas dietas (Tabela 4). O tempo utilizado para a alimentação no cocho está diretamente associado com a quantidade de suplemento concentrado fornecido. Sendo assim, os resultados próximos entre os níveis de glicerina bruta encontrado neste estudo podem ser explicados pelo fornecimento de quantidades iguais de concentrados nas dietas experimentais.

Considerando que as atividades comportamentais são mutuamente excludentes, a ausência de efeito entre os níveis de glicerina nas atividades de tempo de pastejo (PAS), tempo de ruminação (RUM) e tempo de alimentação no cocho (COC) refletiu-se em tempos de outras atividades semelhantes para as cinco dietas.

O tempo de alimentação total (TAT) compreende o somatório entre as atividades de ingestão (PAS e COC), enquanto o tempo de mastigação total (TMT) consiste no conjunto de atividades mastigatórias (PAS, RUM e COC), sendo que o tempo de pastejo (PAS) e tempo de alimentação no cocho (COC) estão relacionados às funções de apreensão da forragem e insalivação do conteúdo de concentrado, e o tempo de ruminação (RUM) para diminuição das partículas ingeridas. Por conseguinte, não havendo diferenças entre os níveis de glicerina bruta para as variáveis PAS, RUM e COC (Tabela 6), também não influíram sobre o tempo de alimentação total (TAT) e o tempo de mastigação total (TMT). 
Tabela 6. Comportamento ingestivo de vacas lactantes mantidas em pastagem tropical com níveis de inclusão de glicerina bruta na dieta, com seu respectivo coeficiente de variação (CV, \%), equação de regressão (ER) e coeficiente de determinação $\left(\mathrm{R}^{2}\right)$.

\begin{tabular}{lcccccccc}
\hline \multicolumn{1}{c}{ Item } & \multicolumn{9}{c}{ Nível de glicerina bruta na dieta (\%) } & \multirow{2}{*}{ CV } & \multirow{2}{*}{ ER } & \multirow{2}{*}{ R $^{2}$} \\
\hline PAS (min.) & 439 & 453 & 461 & 441 & 435 & 12,8 & $\hat{Y}=446$ & --- \\
OUT (min.) & 472 & 469 & 489 & 508 & 487 & 13,8 & $\hat{Y}=485$ & --- \\
RUM (min.) & 491 & 489 & 463 & 464 & 492 & 14,2 & $\hat{Y}=480$ & --- \\
COC (min.) & 37 & 28 & 27 & 26 & 26 & 43,0 & $\hat{Y}=29$ & --- \\
TAT (min.) & 476 & 482 & 488 & 467 & 460 & 13,0 & $\hat{Y}=475$ & --- \\
TMT (min.) & 967 & 971 & 951 & 932 & 953 & 7,0 & $\hat{Y}=955$ & --- \\
\hline
\end{tabular}

PAS - tempo de pastejo; OUT - tempo de outras atividades; RUM - tempo de ruminação; COC - tempo de alimentação no cocho; TAT - tempo de alimentação total; TMT - tempo de mastigação total.

Fonte: Elaboração dos autores.

A glicerina bruta é um coproduto de menor valor comercial que o milho, principal fonte de energia usada na suplementação alimentar de ruminantes com concentrados. Sendo assim, a semelhança entre as dietas contendo quantidades crescentes de glicerina é desejável, pois a utilização do coproduto não deve comprometer o comportamento dos animais em pastejo, nem tampouco o funcionamento adequando do seu processo digestivo. Por isso, as similaridades encontradas para os parâmetros nutricionais e produtivos nestas dietas comparadas com a que contém somente milho como fonte energética é um importante indicativo da ausência de efeito deletério da inclusão da glicerina bruta nas dietas de vacas leiteiras sob pastejo.

Para o número de períodos de pastejo (NPP), outras atividades (NPO), ruminação (NPR) e alimentação no cocho (NPC) não foram observadas diferenças entre os níveis de GB $(\mathrm{P}>0,05)$ (Tabela 7). Verificou-se que os valores de consumos de MS da forragem foram próximos $(8,52$ a 9,59 kg/dia), os consumos de MS do suplemento foram de 4,65 $\mathrm{kg} /$ dia e os consumos de fibra em detergente neutro de 6,34 a 7,06 kg/dia entre as dietas testadas. A inexistência de efeito significativo para o número de períodos das atividades provavelmente deveu-se ao fato de estarem relacionados à extensão destes períodos, que está associada ao tempo despendido com a atividade.
O tempo despendido por período de pastejo (TPP), outras atividades (TPO) e ruminação (TPR) foram semelhantes entre os tratamentos em estudo $(\mathrm{P}>0,05)$. O tempo por período correspondeu ao quociente entre o tempo gasto na atividade pelo número de períodos na mesma atividade, sendo assim, a justificativa para a semelhança entre as dietas decorreu da ausência de efeito nos tempos e períodos das atividades.

O tempo gasto por período de alimentação no cocho (TPC) apresentou efeito quadrático entre os níveis de glicerina $(\mathrm{P}<0,05)$. Tendo em vista, que os ingredientes mais utilizados na alimentação de vacas leiteiras como o milho grão moído e farelo de soja encontram-se na forma sólida, a característica da glicerina é peculiar, pois é um ingrediente líquido. Portanto, na mistura com os demais ingredientes utilizados no presente estudo (milho grão moído, farelo de soja, fosfato bicálcico, calcário calcítico e sal mineral), a mistura do ingrediente líquido com os demais na forma de sólidos formou um composto mais adensado e menos pulvurulento, o que provavelmente promoveu elevação na velocidade de consumo e consequente diminuição do tempo gasto por período de alimentação no cocho. Porém a partir do nível 16,1\% de glicerina na dieta ocorreu o efeito oposto, quando a mistura tornou-se muito pastosa e mais liquefeita, dificultando o consumo pelo animal e elevando o tempo gasto por período de alimentação no cocho. 
Tabela 7. Períodos discretos do comportamento ingestivo de vacas lactantes mantidas em pastagem tropical com níveis de inclusão de glicerina bruta na dieta, com seu respectivo coeficiente de variação (CV, \%), equação de regressão (ER) e coeficiente de determinação $\left(\mathrm{R}^{2}\right)$.

\begin{tabular}{|c|c|c|c|c|c|c|c|c|}
\hline \multirow{2}{*}{ Item } & \multicolumn{5}{|c|}{ Nível de glicerina bruta na dieta (\%) } & \multirow{2}{*}{$\mathrm{CV}$} & \multirow{2}{*}{ ER } & \multirow{2}{*}{$\mathrm{R}^{2}$} \\
\hline & G0 & $\mathrm{G} 4,5$ & G9 & G13,5 & G18 & & & \\
\hline NPP & 9,4 & 11,2 & 9,5 & 9,9 & 10,0 & 20,8 & $\hat{\mathrm{Y}}=10,0$ & --- \\
\hline NPO & 20,6 & 22,4 & 20,6 & 20,5 & 21,2 & 13,8 & $\hat{\mathrm{Y}}=21,1$ & --- \\
\hline NPR & 14,2 & 15,4 & 14,7 & 14,0 & 14,5 & 16,2 & $\hat{Y}=14,6$ & --- \\
\hline NPC & 2,0 & 2,1 & 2,0 & 2,2 & 2,1 & 18,4 & $\hat{\mathrm{Y}}=2,1$ & --- \\
\hline ТРP (min.) & 50,6 & 42,6 & 49,2 & 46,2 & 44,7 & 28,5 & $\hat{\mathrm{Y}}=46,7$ & --- \\
\hline TPO (min.) & 23,3 & 21,5 & 23,7 & 25,1 & 22,9 & 17,5 & $\hat{\mathrm{Y}}=23,3$ & --- \\
\hline TPR (min.) & 37,8 & 32,2 & 31,9 & 33,4 & 34,4 & 28,2 & $\hat{\mathrm{Y}}=34,0$ & --- \\
\hline TPC (min.) & 18,4 & 13,1 & 13,4 & 11,2 & 11,6 & 31,5 & 1 & 0,90 \\
\hline
\end{tabular}

NPP - número de período de pastejo; NPO - número de período de outras atividades; NPR - número de período de ruminação; NPC - número de período de alimentação no cocho; TPP - tempo por período de pastejo; TPO - tempo por período de outras atividades; TPR - tempo por período de ruminação; TPC - tempo por período de alimentação no cocho.

Fonte: Elaboração dos autores.

A taxa de bocado $(\mathrm{TxB})$ apresentou semelhança entre os tratamentos $(\mathrm{P}>0,05)$ (Tabela 8$)$. A média encontrada no presente estudo ( 0,86 bocados/seg.) está próxima dos valores encontrados por Zanine et al. (2009) que em estudo com vacas girolandas em Brachiaria brizantha que relataram 0,69 bocados/ seg. Independente disso, o acréscimo da glicerina nas dietas não influenciou este parâmetro.

A variável massa de bocado $(\mathrm{MaB})$, tanto em termos de MS ou MO apresentou efeito quadrático com a inclusão da glicerina bruta na dieta $(\mathrm{P}<0,05)$. De acordo com a equação encontrada, o menor valor de MaB em MS é 8,7\%, e em MO 8,65\% de inclusão de glicerina bruta. A explicação deste efeito pode estar relacionada à taxa de passagem, pois um dos fatores físicos que estimulam o consumo em ruminantes é a diminuição do conteúdo alimentar no trato gastrointestinal. Talvez o aumento do nível de glicerina tenha influenciado negativamente a degradabilidade ruminal da fibra (ROGER et al., 1992), ocasionando redução da ingestão com o aumento da participação deste composto nas dietas. As médias de número de bocados entre duas deglutições consecutivas (BDe), o tempo entre duas deglutições (TDe) e o número de bocados por dia (NBD) não apresentaram diferenças entre os níveis de glicerina em estudo. Talvez, devido ao fato de os animais receberem suplementação concentrada, acarretando assim menor necessidade de ingestão da forragem para atendimento da exigência nutricional, reflete-se ao menor número de bocados efetuados ao decorrer do dia.

$\mathrm{O}$ número de mastigações merícicas por bolo (MMB) e o tempo por bolo (TBo) foram semelhantes entre os níveis de glicerina $(\mathrm{P}>0,05)$ (Tabela 9). Essas variáveis podem ser consideradas um detalhamento do tempo de ruminação (RUM), pois a variação deste pode ser provocada pela alteração de uma destas variáveis. Da mesma forma que o efeito encontrado no tempo de ruminação (RUM), a semelhança no consumo de fibra em detergente neutro entre os níveis de glicerina, também proporcionou semelhanças para número de mastigações merícicas por bolo (MMB) e tempo gasto para ruminação de cada bolo (TBo). 
Tabela 8. Aspectos dos bocados do comportamento ingestivo de vacas lactantes mantidas em pastagem tropical com níveis de inclusão de glicerina bruta na dieta, com seu respectivo coeficiente de variação (CV, \%), equação de regressão (ER) e coeficiente de determinação $\left(\mathrm{R}^{2}\right)$.

\begin{tabular}{lcccccccc}
\hline \multirow{2}{*}{ Item } & \multicolumn{9}{c}{ Nível de glicerina bruta na dieta (\%) } & \multirow{2}{*}{ CV } & \multirow{2}{*}{ ER } & \multirow{2}{*}{ R $^{2}$} \\
\cline { 2 - 5 } & G0 & G4,5 & G9 & G13,5 & G18 & & & \\
TxB (n ${ }^{\text {/seg.) }}$ & 0,88 & 0,87 & 0,83 & 0,82 & 0,93 & 12,7 & $\hat{\mathrm{Y}}=0,86$ & --- \\
MaB (g MS) & 0,32 & 0,26 & 0,25 & 0,26 & 0,33 & 18,3 & 1 & 0,99 \\
MaB (g MO) & 0,31 & 0,26 & 0,24 & 0,25 & 0,32 & 18,5 & 2 & 0,99 \\
BDe (n ${ }^{\text {o }}$ & 41,6 & 39,2 & 36,4 & 32,2 & 41,6 & 20,1 & $\hat{\mathrm{Y}}=38,2$ & --- \\
TDe (seg.) & 47,0 & 45,6 & 44,5 & 40,0 & 46,2 & 19,6 & $\hat{\mathrm{Y}}=44,7$ & --- \\
NBD & 30155 & 31276 & 33512 & 33022 & 28748 & 14,4 & $\hat{\mathrm{Y}}=31342$ & --- \\
\hline
\end{tabular}

${ }^{1} \hat{Y}=0,001 x^{2}-0,0174 x+0,3209 ;{ }^{2} \hat{Y}=0,001 x^{2}-0,0173 x+0,313$

TxB - Taxa de bocados; MaB - massa do bocado; $\mathrm{BDe}$ - número de bocados entre duas deglutições consecutivas; TDe - tempo entre duas deglutições; NBD - número de bocados por dia.

Fonte: Elaboração dos autores.

Tabela 9. Aspectos da ruminação do comportamento ingestivo de vacas lactantes mantidas em pastagem tropical com níveis de inclusão de glicerina bruta na dieta, com seu respectivo coeficiente de variação (CV, \%), equação de regressão (ER) e coeficiente de determinação $\left(\mathrm{R}^{2}\right)$.

\begin{tabular}{|c|c|c|c|c|c|c|c|c|}
\hline \multirow{2}{*}{ Item } & \multicolumn{5}{|c|}{ Nível de glicerina bruta na dieta (\%) } & \multirow{2}{*}{$\mathrm{CV}$} & \multirow{2}{*}{ ER } & \multirow{2}{*}{$\mathrm{R}^{2}$} \\
\hline & G0 & $\mathrm{G} 4,5$ & G9 & $\mathrm{G} 13,5$ & G18 & & & \\
\hline $\operatorname{MMB}\left(\mathrm{n}^{\circ}\right)$ & 48,0 & 45,7 & 49,5 & 48,6 & 46,6 & 14,5 & $\hat{\mathrm{Y}}=47,7$ & --- \\
\hline TBo (seg.) & 43,5 & 44,0 & 46,4 & 45,6 & 44,5 & 14,5 & $\hat{\mathrm{Y}}=44,8$ & --- \\
\hline VeM (n\%seg.) & 1,10 & 1,05 & 1,08 & 1,06 & 1,06 & 10,5 & $\hat{\mathrm{Y}}=1,07$ & --- \\
\hline TeM (seg.) & 0,91 & 0,96 & 0,94 & 0,95 & 0,96 & 11,6 & $\hat{\mathrm{Y}}=0,94$ & --- \\
\hline MMnd $\left(n^{\circ}\right)$ & 26907 & 28096 & 26077 & 26523 & 28203 & 16,7 & $\hat{Y}=27161$ & --- \\
\hline $\operatorname{BOL}\left(n^{\circ}\right)$ & 685 & 685 & 613 & 622 & 673 & 17,2 & $\hat{\mathrm{Y}}=656$ & --- \\
\hline
\end{tabular}

MMB - número de mastigações merícicas por bolo; TBo - tempo por bolo ruminado ; VeM - velocidade de mastigação; TeM tempo por mastigação; MMnd - número de mastigações merícicas por dia; BOL - número de bolos ruminados por dia.

Fonte: Elaboração dos autores.

A velocidade de mastigação (VeM), tempo de mastigação (TeM), número de mastigações merícicas por dia (MMnd) e número de bolos por dia (BOL) apresentaram-se semelhantes entre os níveis de glicerina $(\mathrm{P}>0,05)$. Observou-se que variáveis calculadas são dependentes das variáveis que compõe sua equação, sendo assim a semelhança no tempo de ruminação (RUM), no número de mastigações merícicas por bolo (MMB) e no tempo gasto para ruminação de cada bolo (TBo) promoveram semelhanças também nas demais variáveis estudadas (VeM, TeM, MMnd e BOL).

A eficiência de alimentação (Tabela 10) representa a velocidade de ingestão dos nutrientes em função do tempo, acarretando em maior disponibilidade dos mesmos para a digestão. Já a eficiência de ruminação de animais consumindo pastagens está associada ao grau de lignificação que se encontra a mesma, sendo necessário maior tempo de ruminação para pastagem mais lignificada, diminuindo assim a eficiência nutricional.

Não foi verificado efeito para as eficiências de alimentação e ruminação entre os níveis de glicerina na dieta $(\mathrm{P}>0,05)$, sendo possivelmente, consequência do consumo e do tempo gasto nestas atividades. 
Tabela 10. Eficiências do comportamento ingestivo de vacas lactantes mantidas em pastagem tropical com níveis de inclusão de glicerina bruta na dieta, com seu respectivo coeficiente de variação (CV, \%), equação de regressão (ER) e coeficiente de determinação $\left(\mathrm{R}^{2}\right)$.

\begin{tabular}{|c|c|c|c|c|c|c|c|c|}
\hline \multirow{2}{*}{ Item } & \multicolumn{5}{|c|}{ Nível de glicerina bruta na dieta (\%) } & \multirow{2}{*}{$\mathrm{CV}$} & \multirow{2}{*}{ ER } & \multirow{2}{*}{$\mathrm{R}^{2}$} \\
\hline & G0 & G4,5 & G9 & G13,5 & G18 & & & \\
\hline \multicolumn{9}{|c|}{ Eficiências de alimentação (g/min) } \\
\hline EAMS & 29,86 & 27,36 & 26,91 & 28,50 & 31,12 & 13,3 & $\hat{\mathrm{Y}}=28,74$ & --- \\
\hline EAFDN & 14,79 & 13,01 & 12,81 & 13,68 & 15,31 & 14,8 & $\hat{\mathrm{Y}}=13,92$ & --- \\
\hline EANDT & 17,52 & 16,34 & 16,42 & 17,05 & 18,65 & 15,8 & $\hat{\mathrm{Y}}=17,20$ & --- \\
\hline EACNF & 8,17 & 7,93 & 7,77 & 7,98 & 8,33 & 12,6 & $\hat{\mathrm{Y}}=8,03$ & --- \\
\hline EAPB & 4,36 & 4,01 & 3,95 & 4,18 & 4,55 & 13,0 & $\hat{\mathrm{Y}}=4,21$ & --- \\
\hline \multicolumn{9}{|c|}{ Eficiências de ruminação ( $\mathrm{g} / \mathrm{min})$} \\
\hline ERMS & 29,07 & 26,71 & 28,00 & 28,46 & 28,71 & 18,3 & $\hat{\mathrm{Y}}=28,39$ & --- \\
\hline ERFDN & 14,42 & 12,68 & 13,35 & 13,67 & 14,61 & 20,6 & $\hat{Y}=13,75$ & --- \\
\hline
\end{tabular}

EAMS - eficiência de alimentação de matéria seca; EAFDN - eficiência de alimentação de fibra em detergente neutro; EANDT eficiência de alimentação dos nutrientes digestíveis totais; EACNF - eficiência de alimentação de carboidratos não fibrosos; EAPB - eficiência de alimentação de proteína bruta; ERMS - eficiência de ruminação de matéria seca; ERFDN - eficiência de ruminação de fibra em detergente neutro.

Fonte: Elaboração dos autores.

\section{Conclusões}

A inclusão de glicerina bruta na dieta de vacas lactantes em pastagem tropical não promove variações no comportamento ingestivo. A ausência de efeitos negativos relacionados ao uso deste coproduto em substituição ao milho permite a sua utilização em até $18 \%$ de glicerina bruta em dietas para vaca leiteiras sem alterar os parâmetros comportamentais relacionados à ingestão de alimentos.

\section{Referências}

ALLDEN, F.; WHITTAKER, A. M. The determinants of herbage intake by grazing sheep: the interrelationship of factors influencing herbage intake and availability. Australian Journal of Agricultural Research, Victoria, v. 21, p. $755-764,1970$.

ALLEN, M. S. Physical constraints on voluntary intake of forages by ruminants. Journal of Animal Science, Champaign, v. 74, n. 12, p. 3063-3075, 1996.

ASSOCIATION OF OFFICIAL ANALYTICAL CHEMISTS - AOAC. Official methods of analysis. $17^{\text {th }}$ ed. Gaithersburg, MD: AOAC International, 2000.
BAGGIO, C.; CARVALHO, P. C. F.; SILVA, J. L. S.; ANGHINONI, I.; LOPES, M. L. T.; THUROWET. Padrões de deslocamento e captura de forragem por novilhos em pastagem de azevém-anual e aveia-preta manejada sob diferentes alturas em sistema de integração lavoura-pecuária. Revista Brasileira de Zootecnia, Viçosa, v. 38, n. 2, p. 215-222, 2009.

BREMM, C.; ROCHA, M. G.; FREITAS, F. K.; MACARI, S.; ELEJALDE, D. A. G.; ROSO, D. Comportamento ingestivo de novilhas de corte submetidas a estratégias de suplementação em pastagens de aveia e azevém. Revista Brasileira de Zootecnia, Viçosa, v. 37, n. 7, p. 1161-1167, 2008.

BURGER, P. J.; PEREIRA, J. C.; QUEIROZ, A. C.; SILVA, J. F. C.; VALADARES FILHO, S. C.; CECON, P. R.; CASALI, A. D. P. Comportamento ingestivo de bezerros holandeses alimentados com dietas contendo diferentes níveis de concentrado. Revista Brasileira de Zootecnia, Viçosa, v. 29, n. 1, p. 236-242, 2000.

CAMPBELL, A. G. Grazed pastures parameters: I. Pasture dry matter production and availability in a stocking rate and grazing management experiment with dairy cows. Journal of Agricultural Science, Toronto, v. 67, p. 211-216, 1966. 
CASALI, A. O.; DETMANN, E.; VALADARES FILHO, S. C.; PEREIRA, J. C.; HENRIQUES, L. T. FREITAS, S. G.; PAULINO, M. F. Influência do tempo de incubação e do tamanho de partículas sobre os teores de compostos indigestíveis em alimentos e fezes bovinas obtidos por procedimentos in situ. Revista Brasileira de Zootecnia, Viçosa, v. 37, n. 2, p. 335-342, 2008.

FUKUMOTO, N. M.; DAMASCENO, J. C.; DERESZ, F.; MARTINS, C. E.; COSER, A. C.; SANTOS, G. T. Produção e composição do leite, consumo de matéria seca e taxa de lotação em pastagens de gramíneas tropicais manejadas sob lotação rotacionada. Revista Brasileira de Zootecnia, Viçosa, v. 39, n. 7, p. 1548-1557, 2010.

GARDNER, A. L. Técnicas de pesquisa em pastagem e aplicabilidade de resultados em sistema de produção. Brasília: IICA/EMBRAPA/CNPGL, 1986. 197 p.

GARY, L. A.; SHERRITT, G. W.; HALE, E. B. Behavior of charolais cattle on pasture. Journal of Dairy Science, Champaign, v. 30, n. 2, p. 303-306, 1970.

HALL, M. B. Challenges with non-fiber carbohydrate methods. Journal of Animal Science, Champaign, v. 81, n. 12, p. 3226-3232, 2003.

HODGSON, J. Ingestive behavior. In: LEAVER, J. D. (Ed.). Herbage intake handbook. Hurley: British Grassland Society, 1982. 113 p.

ÍTAVO, L. C. V.; SOUZA, S. R. M. B. O.; RÍMOLI, J.; ÍTAVO, C. C. B. F.; DIAS, A. M. Comportamento ingestivo diurno de bovinos em pastejo contínuo e rotacionado. Archivos de Zootecnia, Cordoba, v. 57, n. 217, p. 43-52, 2008.

JAMIESON, W. S.; HODGSON, J. The effect of variation in sward characteristics upon the ingestive behavior and herbage intake of calves and lambs under continuous stocking management. Grass and Forage Science, Malden, v. 34, n. 4, p. 273-281, 1979.

JOHNSON, A. D. Sample preparation and chemical analysis of vegetation. In: MANNETJE, L. (Ed.). Measurement of grassland vegetation and animal production. Aberystwyth: Commonwealth Agricultural Bureaux, 1978. p. 96-102.

MACARI, S.; ROCHA, M. G.; POTTER, L.; ROMAN, J.; BREMM, C.; COSTA, V. G. Comportamento ingestivo diurno de novilhas de corte recebendo níveis de suplemento. Ciência Rural, Santa Maria, v. 37, n. 6, p. 1746-1752, 2007.

McMENIMAN, N. P. Methods of estimating intake of grazing animals. In: REUNIÃO ANUAL DA
SOCIEDADE BRASILEIRA DE ZOOTECNIA, SIMPÓSIO SOBRE TÓPICOS ESPECIAIS EM ZOOTECNIA, 34., 1997, Juiz de Fora. Anais... Juiz de Fora: Sociedade Brasileira de Zootecnia, Juiz de Fora, 1997. p. 131-168.

MISSIO, R. L.; BRONDANI, I. L.; ALVES FILHO, D. C.; SILVEIRA, M. F.; FREITAS, L. S.; RESTLE, J. Comportamento ingestivo de tourinhos terminados em confinamento, alimentados com diferentes níveis de concentrado na dieta. Revista Brasileira de Zootecnia, Viçosa, v. 39, n. 7, p. 1571-1578, 2010.

NATIONAL RESEARCH COUNCIL - NRC. Nutrient requirements of dairy cattle. 7. ed. Washington: National Research Council, 2001.381 p.

NUNES, A. S.; OLIVEIRA, R. L.; AYRES, M. C. C.; BAGALDO, A. R.; GARCEZ NETO, A. F.; BARBOSA, L. P. Condição hepática de cordeiros mantidos com dietas contendo torta de dendê proveniente da produção de biodiesel. Revista Brasileira de Zootecnia, Viçosa, v. 39, n. 8, p. 1825-1831, 2010.

OSBORNE, V. R.; ODONGO, N. E.; CANT, J. P.; SWANSON, K. C.; McBRIDE, B. W. Effects of supplementing glycerol and soybean oil in drinking water on feed and water intake, energy balance, and production performance dairy cows. Journal of Dairy Science, Champaign, v. 92, n. 2, p. 698-707, 2009.

PALHANO, A. L.; CARVALHO, P. C. F.; DITTRICH, J. R.; MORAES, A.; SILVA, S. C.; MONTEIRO, A. L. G. Características do processo de ingestão de forragem por novilhas holandesas em pastagens de capim-mombaça. Revista Brasileira de Zootecnia, Viçosa, v. 36, n. 4, p. 1014-1021, 2007.

PARDO, R. M. P.; FISCHER, V.; BALBINOTTI, M.; ZANELA, M. B.; MORENO, C. B.; FERREIRA, E. X.; VINHAS, R. I.; MONKS, P. L. Comportamento ingestivo diurno de novilhos em pastejo a níveis crescentes de suplementação energética. Revista Brasileira de Zootecnia, Viçosa, v. 32, n. 6, p. 1408-1418, 2003.

PAULINO, M. F.; DETMANN, E.; VALADARES FILHO, S. C. Suplementação animal em pasto: energética ou protéica?. In: SIMPÓSIO SOBRE MANEJO ESTRATÉGICO DA PASTAGEM, 3., 2006, Viçosa, MG. Anais... Viçosa, MG: SIMFOR, 2006. p. 359-392.

PEREIRA. E. S.; PIMENTEL, P. G.; CARNEIRO, M. S. S.; MIZUBUTI, I. Y.; RIBEIRO, E. L. A.; ROCHA JUNIOR, J. N.; COSTA, M. R. G. F. Comportamento ingestivo de vacas em lactação alimentadas com ração a base de torta de girassol. Semina: Ciências Agrárias, Londrina, v. 32, n. 3, p. 1201-1210. 2011. 
REGO, F. C. A.; DAMASCENO, J. C.; FUKUMOTO, N. M.; CORTES, C.; HOESHI, L.; MARTINS, E. N.; CECATO, U. Comportamento ingestivo de novilhos mestiços em pastagens tropicais manejadas em diferentes alturas. Revista Brasileira de Zootecnia, Viçosa, v. 35, n. 4, p. 1611-1620, 2006.

ROGER, V.; FONTY, G.; ANDRE, C.; GOUET, P. Effects of glycerol on the growth, adhesion and cellulotytic activity of rumen cellulolytic bacteria and anaerobic fungi. Current Microbiology, v. 25, n. 4, p. 197-201, 1992.

SANTANA JÚNIOR, H. A.; FIGUEIREDO, M. P.; CARDOSO, E. O.; MENDES, F. B. L.; ABREU FILHO, G.; PINHEIRO, A. A.; VIANA, P. T.; ROSEIRA, J. P. S. Crude glycerin in supplement to primiparous lactating cows grazing on tropical pasture: nutritional and productive characteristics. Revista Brasileira de Zootecnia, Viçosa, v. 42, n. 2, p. 117-124, 2013a.

SANTANA JÚNIOR, H. A.; SILVA, R. R.; CARVALHO, G. G. P.; CARDOSO, E. O.; MENDES, F. B. L.; PINHEIRO, A. A.; ABREU FILHO, G.; DIAS, D. L. S.; BARROSO, D. S.; SILVA, F. F.; TRINDADE JÚNIOR, G. Comportamento ingestivo de novilhas suplementadas a pasto sob nutrição compensatória. Archivos de Zootecnia, Cordoba, v. 62, n. 237, p. 61-71, 2013b.

SANTANA JÚNIOR, H. A.; SILVA, R. R.; CARVALHO, G. G. P.; SILVA, F. F.; BARROSO, D. S.; PINHEIRO, A. A.; ABREU FILHO, G.; CARDOSO, E. O.; DIAS, D. L. S.; TRINDADE JÚNIOR, G. Correlação entre desempenho e comportamento ingestivo de novilhas suplementadas a pasto. Semina: Ciências Agrárias, Londrina, v. 34, n. 1, p. 367-376, $2013 \mathrm{c}$.

SANTOS, E. M.; ZANINE, A. M.; PARENTE, H. N.; FERREIRA, D. J.; ALMEIDA, J. C. C. Comportamento ingestivo de bezerras (Holandês x Zebu) sob pastejo no cerrado goiano. Ciência Animal Brasileira, Goiânia, v. 7, n. 2, p. 143-151, 2006.
SNIFFEN, C. J.; O'CONNOR, D. J.; Van SOEST, P. J.; FOX, D. G.; RUSSEL, J. B. A net carbohydrate and protein system for evaluating cattle diets: carbohydrate and protein availability. Journal of Animal Science, Champaign, v. 70, n. 12, p. 3562-3577, 1992.

TEIXEIRA, F. A.; BONOMO, P.; PIRES, A. J. V.; SILVA, F. F.; MARQUES, J. A.; SANTANA JUNIOR, H. A. Padrões de deslocamento e permanência de bovinos em pastos de Brachiaria decumbens diferidos sob quatro estratégias de adubação. Revista Brasileira de Zootecnia, Viçosa, v. 40, n. 7, p. 1489-1496, 2011.

TEIXEIRA, F. A.; MARQUES, J. A.; SILVA, F. F.; PIRES, A. J. V. Comportamento ingestivo e padrão de deslocamento de bovinos em pastagens tropicais. Archivos de Zootenia, Cordoba, v. 59, p. 57-70, 2010.

THOMPSON, J. C.; HE, B. B. Characterization of crude glycerol from biodiesel production from multiple feedstoks. Applied Engineering in Agriculture, St. Joseph, v. 22, n. 2, p. 261-265, 2006.

Van SOEST, P. J.; ROBERTSON, J. B.; LEWIS, B. A. Methods for dietary fiber, neutral detergent fiber and nonstarch polysaccharides in relation to animal nutrition. Journal of Dairy Science, Champaign, v. 74, n. 10, p. 3583-3597, 1991.

WEISS, W. P. Energy prediction equations for ruminant feeds. In: CORNELL NUTRITION CONFERENCE FOR FEED MANUFACTURERS, 61., 1999, Ithaca. Proceedings... Ithaca: Cornell University, 1999. p. 176185.

WILM, H. G.; COSTELlO, D. F.; KLIPPLE, G. E. Estimating forage yield by the double sampling method. Journal of American Society of Agronomy, Madison, v. 36, n. 3, p. 194-203, 1994.

ZANINE, A. M.; VIEIRA, B. R.; FERREIRA, D. J.; VIEIRA, A. J. M.; LANA, R. P.; CECON, P. R. Comportamento ingestivo de vacas Girolandas em pastejo de Brachiaria brizantha e Coast-cross. Revista Brasileira de Saúde e Produção Animal, Salvador, v. 10, n. 1, p. 85-95, 2009. 
